\title{
HETEROKARYON COMPATIBILITY OF UNRELATED STRAINS IN THE ASPERGILLUS NIDULANS GROUP
}

\author{
M. GRINDLE \\ A.R.C. Unit of Biometrical Genetics, Deportment of Genetics, \\ University of Birmingham
}

Received I 4.xi.6z

\section{INTRODUCTION}

Heterokaryosis, the occurrence of genetically different nuclei within a common cytoplasm, is not only a system of somatic flexibility in wild fungi (Jinks, $1952 b$ ) but it is also an essential first step in a number of processes which lead to the recombination of nuclear genes and reassortment of cytoplasmic differences in fungi. For example, it is a necessary preliminary to the parasexual cycle in both sexual and imperfect fungi and to outcrossing in homothallic species (see Pontecorvo, 1956 and Roper, 1962 for reviews). The formation of a heterokaryon between different strains involves anastomosis of their hyphæ so that both the nuclei and the cytoplasms of the strains are brought together in the same cell lineage. Thus, in those species having no other mechanism for the transfer of genetic material from one strain to another, the ability of different strains to form heterokaryons, that is, their heterokaryon compatibility determines whether heritable differences, transmitted by the nucleus or the cytoplasm, can be exchanged. Consequently, it may indicate whether variation within such species arises by recombination and reassortment rather than by direct mutation alone.

The present paper is the first of a series in which the heterokaryon compatibility of a number of wild strains of Aspergillus nidulans and allied species will be described and related to the morphology and geographical derivation of the isolates involved. A. nidulans has been chosen as the test organism because it is known to form heterokaryons and undergo both sexual and parasexual recombination in the laboratory (Pontecorvo, r 953).

\section{MATERIALS AND METHODS}

Twenty-five isolates, most of them $A$. nidulans, were obtained from a variety of sources. Relevant information on their origins is included in the list of strains given in table $\mathrm{I}$. Strains acquired from culture collections are named and described in accordance with the information given in the appropriate culture lists ; those from British universities are given the designations supplied by the donors; Birmingham isolates were identified from IMI type culture 16040 and both strains of $A$. nidulans var. echinulatus were identified by $\mathrm{Mr} \mathrm{J}$. J. Elphick of the CMI staff.

All isolates were maintained in the laboratory at $25^{\circ} \mathrm{C}$. on Czapek minimal medium (MM) containing New Zealand agar supplied by G. T. Gurr Ltd. of London. 
Auxotrophs were maintained on the complete medium (CM) described by Pontecorvo (1953).

To obtain measurements of growth-rate, sporulation and sexuality, all cultures were grown on $20 \mathrm{ml}$. MM in $9 \mathrm{~cm}$. Petri dishes at $25^{\circ} \mathrm{C}$. Each growth-rate

TABLE I

List of strains

(CMI = IMI = Commonwealth Mycological Instit., Kew; CBS = Centraalbureau voor Schimmelcultures, Baarn ; NRRL $=$ Northern Utilisation Research Branch, U.S.D.A., U.S.A.)

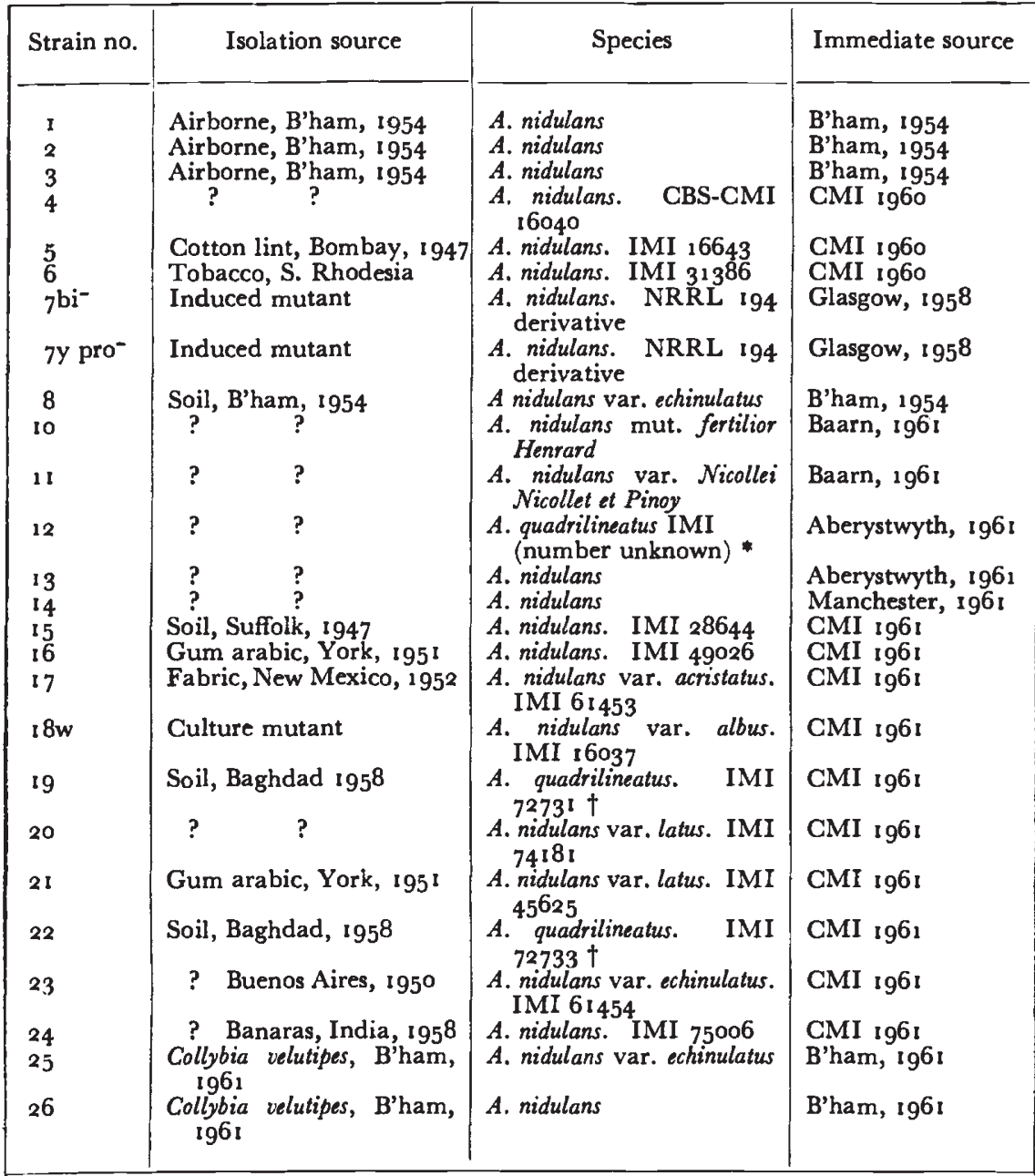

* Received from CMI as strain of $A$. nidulans.

$\uparrow$ Originally catalogued as $A$. nidulans.

measurement indicates the average daily increase in colony diameter of 4 replicate colonies as measured from the 3-8 days after inoculation. Estimates of perithecial density were obtained by marking circles of $7 \mathrm{~mm}$. diam. on each of 3 replicate 28-day-old colonies and counting the number of mature perithecia (sexual reproductive structures) in them; with each colony, 2 circles were taken near the inoculum and 2 near the growing margin. The same 12 circles were transferred 
into measured amounts of Tween 8o (polyoxyethylene sorbitan monooleate, a wetting agent) and the number of conidia (asexual spores) in the suspensions were estimated from haemocytometer counts.

Spore-colourm utants were obtained by ultraviolet irradiation of wild-type conidia suspended in 1 per cent. physiological saline. Irradiated samples were plated on Plunkett's medium (Plunkett, 1953) of $p \mathrm{H} 5.2$ at a density of about $10^{6}$ per Petri dish and examined after 4 days for white or yellow conidial heads.

The Plunkett medium (PM) used has the following composition; sucrose, $50.0 \mathrm{gm} . ;$ dipotassium hydrogen phosphate, $5^{\circ} \mathrm{o} \mathrm{gm} . ;$ magnesium sulphate, $2.5 \mathrm{gm}$; asparagine, $\mathrm{I} \cdot 0 \mathrm{gm}$. ; thiamine, $50 \mu \mathrm{gm}$.; ferrous sulphate, trace ; New Zealand agar, $15 \mathrm{gm}$.; distilled water, rooo $\mathrm{ml}$ The $p \mathrm{H}$ is adjusted with I. $\mathrm{MM}$ hydrochloric acid.

\section{RESULTS}

(i) Morphological differences between isolates

The main morphological characters of each strain are listed in table 2. In addition to the three comparative criteria used other

\section{TABLE 2}

Metrical characteristics of strains grown on $M M$ and sporulation on $P M$, $\mathrm{pH} 5.2$ measured on an arbitrary scale from $\mathrm{I}-6$

\begin{tabular}{|c|c|c|c|c|}
\hline Strain & $\begin{array}{l}\text { Growth-rate } \\
\mathrm{mm} \text {. per day }\end{array}$ & $\begin{array}{c}\text { No. conidia } \\
\text { per } \mathrm{mm}^{2} \times 10^{-3}\end{array}$ & $\begin{array}{l}\text { No. perithecia } \\
\text { per } \mathrm{mm}^{2}\end{array}$ & $\begin{array}{c}\text { Sporulation } \\
\text { on } \mathrm{PM}, p \mathrm{H}_{5} \cdot 2\end{array}$ \\
\hline 1 & $5 \cdot 1$ & $3^{6 \cdot 3}$ & 0.1 & $I$ \\
\hline $1 w_{3}$ & $4 \cdot 8$ & $22 \cdot 9$ & $1 \cdot 7$ & I \\
\hline 2 & $5 \cdot 0$ & $28 \cdot 0$ & $0 \cdot 1$ & I \\
\hline $2 w$ & $4 \cdot 8$ & $21 \cdot 0$ & 0.7 & $I$ \\
\hline 3 & $5 \cdot 7$ & $16 \cdot 6$ & 0.1 & 1 \\
\hline 4 & $3 \cdot 7$ & 53.4 & 0.0 & I \\
\hline $4 \mathrm{w}$ & $3 \cdot 6$ & $27 \cdot 4$ & $I \cdot 2$ & I \\
\hline 5 & $1 \cdot 6$ & $3^{2} \cdot 3$ & 0.8 & 2 \\
\hline $5 w$ & $2 \cdot 2$ & I $7 \cdot 3$ & 0.5 & 2 \\
\hline$\overline{6}$ & $4 \cdot 8$ & $45 \cdot 3$ & $\mathrm{I} \cdot 2$ & I \\
\hline $6 w$ & $4 \cdot 5$ & $39^{\circ} 7$ & 0.8 & I \\
\hline 8 & $3 \cdot 4$ & $2 \cdot 0$ & 0.8 & 2 \\
\hline 10 & $4 \cdot 2$ & 20.4 & I.9 & I \\
\hline row & 3.9 & $17 \cdot 3$ & $I \cdot O$ & I \\
\hline 11 & $4 \cdot 4$ & $8 \cdot 1$ & $0 \cdot 1$ & I \\
\hline 11y & $3 \cdot 9$ & $6 \cdot 8$ & 0.2 & I \\
\hline 12 & 4.6 & $6 \cdot I$ & $4^{\cdot 2}$ & I \\
\hline I $2 y$ & $4 \cdot 8$ & $4^{\cdot I}$ & $4 \cdot 0$ & I \\
\hline 13 & 4.9 & $39 \cdot 7$ & I. 4 & I \\
\hline $13 w$ & $4 \cdot 7$ & $27 \cdot I$ & $I \cdot 2$ & I \\
\hline I 4 & 50 & $20 \cdot 2$ & $I \cdot O$ & I \\
\hline $14 y$ & $4 \cdot 6$ & 19.0 & I. 5 & I \\
\hline 15 & $2 \cdot 5$ & I. 8 & 0.1 & 6 \\
\hline 16 & $I \cdot I$ & 0.0 & $2 \cdot 5$ & 6 \\
\hline 17 & $3 \cdot 4$ & 0.3 & $1 \cdot 2$ & 6 \\
\hline $18 w$ & $5 \cdot 9$ & 19.3 & 0.0 & I \\
\hline 19 & $4 \cdot 7$ & 2.4 & $4 \cdot 5$ & I \\
\hline 20 & 4.7 & $1 \cdot 0$ & 1.5 & 3 \\
\hline 21 & 5.6 & $1 \cdot 3$ & $2 \cdot 0$ & 3 \\
\hline 22 & $4 \cdot 5$ & $6 \cdot 5$ & $7 \cdot 1$ & 1 \\
\hline 23 & 3.0 & 0.0 & 0.2 & 4 \\
\hline 24 & $3 \cdot 9$ & 0.0 & 0.6 & 5 \\
\hline 25 & 3.2 & 3.0 & 0.9 & 2 \\
\hline 26 & $5 \cdot 4$ & $3^{8 \cdot 7}$ & 0.8 & 1 \\
\hline $26 y$ & 5.5 & $4^{8 \cdot 4}$ & 0.7 & 1 \\
\hline
\end{tabular}


distinguishing features, including conidiophore size and shape, intensity of spore colouration and pigmentation both within the hyphæ and secreted into the medium were obvious. Certain groups of strains were either identical or basically similar in appearance but other isolates were unique. Some of these similarities and differences are outlined below.

Strains I, 2, 3, 6, I0, I3, I4 and 26 : basically similar to type culture IMI I6040.

Strains I, 2 and 3 : very similar in appearance but slight differences in surface morphology.

Strains 4 and Io: colonies identical in appearance.

Strains 8 and 25: colonies identical in appearance.

Strains I2, I 9 and 22 : colonies identical in appearance.

Strain 5: large conidial heads; much more aerial mycelium produced than by all other strains.

Strain 18 : very small conidial heads.

Strain I5: colonies yellow; yellow pigment secreted.

Strain I6 : colonies covered entirely by large perithecia.

Although the differences between our isolates of $A$. nidulans were large and indicated a very variable species, it is difficult to assess how much of this variation would have been apparent if all strains had been freshly isolated from the wild. Some strains had been maintained in culture collections for many years on a variety of media, MM, malt agar or PDA (potato dextrose agar), and some had been preserved under mineral oil. The effects of successive propagations by mass hyphal transfer, of different media and of mineral oil must have led to an accumulation of nuclear gene mutations in many isolates and for these reasons it was not possible to make any meaningful correlations between morphological characters of these strains and their isolation source. Further work, however, involving only freshly isolated material from England is now in progress and all of the is isolates tested so far are basically similar in appearance to type culture IMI I6040. Nevertheless, they can be distinguished on various morphological and physiological differences particularly in sporulation, sexuality, pigmentation and rate of growth.

\section{(ii) Effect of different media on growth and sporulation}

One of the requirements for the heterokaryon tests is a medium which promotes maximum sporulation of the strains, so a number of available media were compared using isolates I, 8, I7 and 22 as tests strains (plate I, figs. I-4). Maximum production of conidia was obtained using $\mathrm{PM}\left(p \mathrm{H}_{4} \cdot 6-5 \cdot 2\right)$ followed by $\mathrm{PM}(p \mathrm{H} \mathrm{6 \cdot 0-7 \cdot 0})$, 2 per cent. malt agar $\left(p \mathrm{H}_{5} \cdot 4\right), \mathrm{PM}\left(p \mathrm{H}_{3 \cdot 2-4 \cdot 4}\right), 2$ per cent. malt agar $\left(p \mathrm{H}_{4} \cdot \mathrm{o}\right)$, CM, MM $\left(p \mathrm{H}_{5} \cdot 8\right)$ or MM with additional phosphate and sucrose, PDA (4 per cent. dextrose) and MM ( $p \mathrm{H} 7 \cdot 0)$. On PM $\left(p \mathrm{H}_{4} \cdot 6-5 \cdot 2\right)$ isolates $\mathrm{I}, 2,3,4,5,6,8$, I , I I, I 2, I 3, I 4, I 8, I 9, 22, 25 and 26 were almost completely conidial (plate I, fig. 3). Malt 
agar $\left(p \mathrm{H}_{5} .4\right)$ gave similar results except that the strains were more sexual and conidiophores were spaced further apart (plate I, fig. 6). Sporulation of some strains was only slightly better on these two media than on MM and others remained aconidial regardless of changes in media or culture conditions (e.g. strain I 7, plate I, figs. I-4).

Lowering the $p \mathrm{H}$ of any medium increased sporulation and also affected growth-rate, pigmentation and spore-colour so that many strains easily distinguishable on MM looked identical, especially when grown on PM or 2 per cent. malt agar. Optimal $p \mathrm{H}$ for growth on any medium varied both between isolates and between the sporecolour mutants and the wild-type of the same isolate.

These tests showed that the medium most suitable for distinguishing between isolates is MM containing New Zealand agar. Even the use of Oxoid No. 3 (Spanish) agar in place of N.Z. agar in MM increased growth-rate and sporulation and often affected pigmentation.

\section{HETEROKARYON TESTS}

Proof of heterokaryosis can be supplied by the formation of a "balanced heterokaryon" whose growth is an improvement on the two homokaryons from which it was obtained. Typical examples of "balanced heterokaryons" are those in which (a) growth on any medium is normal whereas each homokaryon grows poorly on the same medium or $(b)$ growth on $\mathrm{MM}$ is possible whereas neither homokaryon, each having a different nutritional requirement, can grow at all on MM. In contrast, where naturally occurring heterokaryons have been obtained from the wild, some component homokaryons have been found to differ very little indeed in rate of growth (Jinks, I $952 b$ ). Both of these proofs, however, require mutant strains whose derivation is difficult and time consuming. Even more important, by using biochemically deficient mutants a heavy selection pressure is applied which favours heterokaryon formation and this may give a false indication of the potentiality of naturally occurring strains to heterokaryotise under natural conditions.

A simpler method for detecting heterokaryons in $A$. nidulans requires only that the two component homokaryons differ by major genes affecting spore-colour. If two parental strains have green spores and white spores respectively then a heterokaryotic mycelium formed after hyphæ of the homokaryons have anastomosed will contain nuclei from both green-spored and white-spored parents. During conidiophore formation in such a heterokaryon, both types of nucleus may be present in each vesicle but each sterigma, from which chains of conidia arise, is homokaryotic. Hence, all conidia in any one chain have the same nuclear type and will be either green or white, but in any single conidial head both green and white spores will be present. Thus, the production of mixed conidial heads (plate II, figs. $4^{-6}$ ) by a culture consisting of two homokaryons differing in 
respect of spore colouration indicates that the two strains are heterokaryon compatible. By this method, even when a mixed culture consists almost entirely of homokaryotic hyphæ, the presence of a few heterokaryons can be detected despite the fact that they are not at a selective advantage in rate of growth. Observation of hyphal anastomosis has been used as a method of testing strains (Ishitani and Sakaguchi, I956) but this is, in itself, probably an unreliable criterion of heterokaryon compatibility since it has been shown in Neurospora crassa that hyphal fusion occurs readily between noncompatible strains (Wilson et al., I g6I).

Spore colour mutants were obtained in the way already described. With most strains, as many as 24 independent mutations resulting in a non-green spore colour were obtained in each $10^{6}$ irradiated spores plated, but in each of isolates I and 2 only one spore-colour mutant (white) was recovered from more than ten times as many spores. No spore-colour mutants at all of strain 3 have been observed on the 24 plates examined. Altogether, slightly more yellow than white spore-colour mutants were seen but in nearly all strains both colours were common. Most non-white colour variants had deep yellow spores but pale yellow, pale green (grey) and olive green conidial heads also occurred in small numbers.

When testing any two isolates for heterokaryon compatibility, the green-spored wild-types and their spore-colour variants were inoculated together in all combinations involving both green and nongreen conidia. For each test, both $\mathrm{PM}\left(p \mathrm{H}_{5} \cdot 2\right)$ and 2 per cent. malt agar $\left(p \mathrm{H}_{5} .4\right)$ were used (plate I, figs. 5 and 6 ) except where auxotrophic strains were involved in which case CM was used instead. To estimate the frequency of mixed conidial heads in a mixed culture, several areas of $2.5 \mathrm{sq}$. mm. in which both parental conidial types were intermingled (plate II, figs. I -3) were selected at random. These were examined at Ioo $\times$ magnification (plate II, fig. 3) and the number of mixed conidial heads (plate II, figs. 4-6) was determined as a proportion of the total number of conidial heads in the field. The mean frequency of mixed heads in a number of different areas from each of at least three mixed cultures was taken as an indication of the degree of heterokaryon compatibility of any two strains (table 3). Numerous factors are known to affect hyphal anastomosis (Raper and Fennell, I953; Ishitani and Sakaguchi, r956) and these would, in turn, affect the frequencies of mixed conidial heads. However, since mixed heads were produced readily on PM $\left(p \mathrm{H}_{5} \cdot 2\right)$ and only slightly less so on 2 per cent. malt agar $\left(p \mathrm{H}_{5} .4\right)$ these two media were used in parallel and under controlled conditions of environment $\left(25^{\circ} \mathrm{C}\right.$.) and $p \mathrm{H}$ (any $p \mathrm{H}$ below 5.0 greatly reduced the proportion of mixed heads; a $p \mathrm{H}$ of about $5 \cdot 6-6 \cdot 2$ seemed optimal for heterokaryon formation, giving a slight increase in mixed heads, but sporulation of many strains at these $p H$ 's was poor).

The results of these tests, summarised in table 3 , show that all 


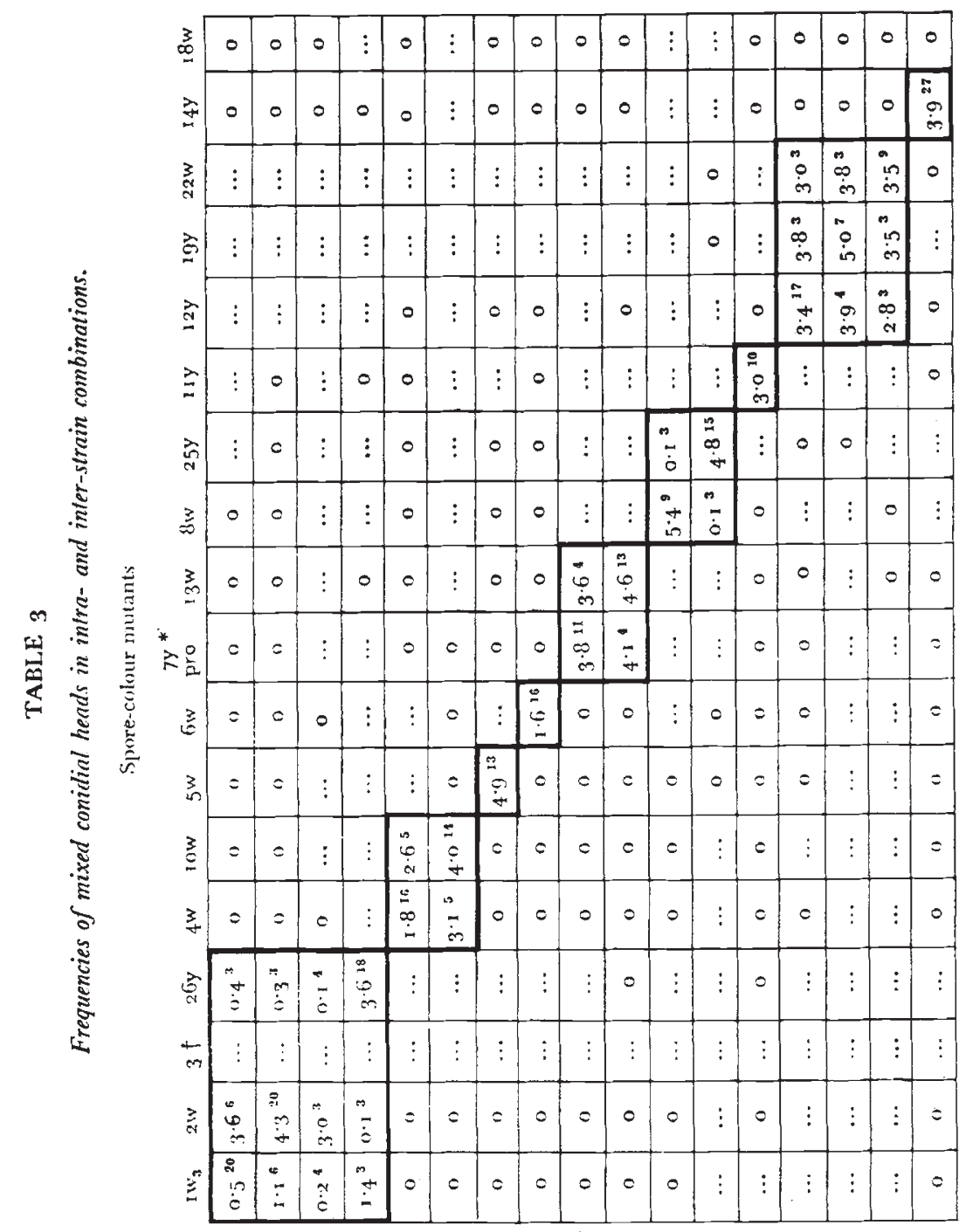

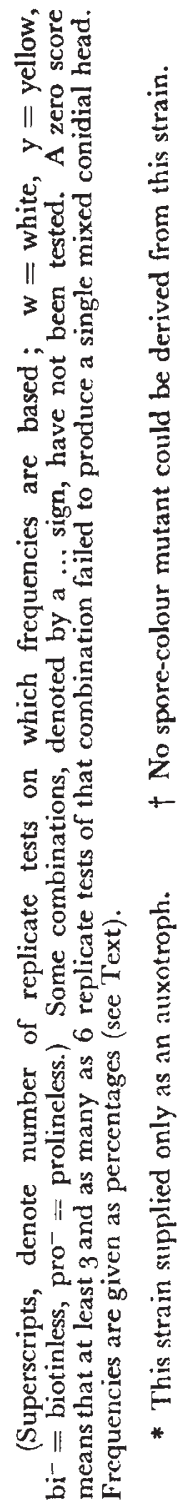


wild type isolates are compatible with their own respective sporecolour mutants but few examples of inter-strain, and none of interspecies, compatibility were found. Furthermore, the examples of inter-strain compatibility involved isolates which are either believed or are known to have a common source and should, therefore, be classed as identical or near-identical strains. Strains 7 and 13 both came originally from Glasgow; strains I9 and 22 were recovered from soil samples taken from the same locality and sent together to the CMI, and strain 12 is probably a subculture of one of these since it came originally from CMI and is, like 19 and 22, a strain of $A$. quadrilineatus (a member of the $A$. nidulans group). Isolates 4 and ro are ex-Baarn strains and they produce colonies which are identical in appearance; it is suggested, therefore, that Henrard's mutant (isolate Io) is a derivative of isolate 4 or it is perhaps the same strain. This leaves only the two isolates of $A$. nidulans var. echinulatus and four isolates of $A$. nidulans in which compatibility of different strains is reasonably certain. All of these were isolated in the same area, Birmingham.

\section{DISCUSSION}

\section{(i) Use of heterokaryon tests for determining species relationships}

Although every one of our wild-type strains formed heterokaryons readily with their own spore-colour variants very few of them were compatible with different strains of the same species and in all examples of inter-strain compatibility, mixed heads were much less common than in comparable intra-strain combinations. Estimates of the frequencies with which different strains heterokaryotise may, therefore, be a measure of the genic similarity of those strains. Thus, where the frequencies of mixed conidial heads both within and between strains are almost identical ( 2 and $3 ; 4$ and Io ; 12, 19 and 22) the strains concerned differ probably by only a few genes. In other instances, where strains form heterokaryons more rarely with each other than they do with their own spore-colour mutants (2 or 3 and $26 ; 8$ and 25) the differences in genotype are presumably more extensive. On this assumption, strain $18 w$ which arose spontaneously from the wildtype strain NRRL.I94 should have been compatible with strains 7 and 13 ; their failure to form heterokaryons is presumed to be due to an accumulation of gene mutations in the white spored strain, I $8 w$, since it first arose because strains $13 \mathrm{w}$ and $\mathrm{r} 8 \mathrm{w}$ differ substantially in many respects (see section 3 (i)).

The nature and number of genic, and perhaps also cytoplasmic, differences required to prevent, or reduce the frequency of, heterokaryon formation between strains is difficult to assess. Heterokaryosis between strains of Fusarium oxysporum (Buxton and Ward, I962) and strains of Aspergillus fonsecaeus (Raper and Fennell, I953) seems to be affected by the nature of the nuclear mutations induced in the parent homokaryons, and even single gene differences appear to determine 
the presence or absence of heterokaryons. On the other hand, numerous derivatives of strain NRRL I 94 of $A$. nidulans, differing by a large number of major gene mutations which arose in the laboratory, are compatible (Kafer, I958) although the frequencies of mixed heads and ease of heterokaryon formation in the various crosses have not been compared. Recent work with English isolates of $A$. nidulans (Grindle, unpublished) has shown that there are many "compatibility groups" within which heterokaryons are formed easily but between these groups, heterokaryons occur with a much reduced frequency or not at all. It seems, therefore, that a complex system of incompatibility factors is responsible.

Initial work on English strains suggests that they are $(a)$ very similar in appearance but can still be distinguished on a number of morphological characters, and $(b)$ sometimes inter-strain compatible, but not always so since even isolates from the same soil sample may be incompatible. The work of Jinks $(1952 a)$ indicates that a similar situation exists among English strains of the Asymmetrica group of the genus Penicillium. He found heterokaryon compatibility between some, but not all, wild isolates and observed that although his isolates were similar in appearance, they could usually be distinguished on a number of morphological and physiological characters.

The ability of different strains to form heterokaryons may prove to be clinal. If so, gene flow within localised populations will be relatively unrestricted but between two spatially isolated populations it will be either interrupted or occur only gradually through other intermediary compatible strains. It is hoped that further work now in progress will show whether such gradients or clines exist in English populations of $A$. nidulans, and also reveal the nature of the incompatibility system.

The application of heterokaryon compatibility tests as an aid in fungal taxonomy and in determining species relationships is limited technically by the necessity for good sporulation media and sporecolour mutants (two different colours may be required in each strain for species with multinucleate spores) or other marked strains (morphological or biochemical-requiring mutants). As in Taschdjian and Muskatblitt's (1955) proposed system of hyphal fusion analysis, most strains, even though similar in appearance, are not compatible so the absence of heterokaryons may not imply a species difference; the issue is complicated further when both hyphal and mating type incompatibility systems are superimposed. Conversely, compatibility of different isolates may be evidence for their close relationship so the heterokaryon technique should be useful particularly in assessing the genic similarity of morphological variants and physiological races of the same species-it has already been shown in the case of the Birmingham isolates that morphological similarities are, by themselves, not necessarily reliable indications of the degree of relationship between strains. By using mutant strains with nutritional requirements, 
genetic relationships have been shown between formæ of Fusarium oxysporum (Garber et al., I $96 \mathrm{I}$ ), between formæ of $F$. oxysporum and Gibberella fujikuroi (Dhillon et al., I96I) and between formæ of $F$. oxypsorum and $F$. solani and Nectria hematococca (Buxton and Ward, I 962 ).

\section{(ii) Validity of different criteria for detecting heterokaryons of} filamentous fungi

The evidence for heterokaryosis as presented by various workers is of two main types, cytological and genetical. Cytological criteria are seldom used, however, and where they involve the observation of anatomosis between hyphæ of different parents the tests are unreliable for the reasons outlined in section 3 .

Genetical evidence depends on two basic phenomena, segregation and complementation.

I. Segregation. (a) In species with uninucleate spores, heterokaryons derived from two homokaryotic strains which differ in the colour of their asexual spores can be detected if they produce conidiophores in which both parental spore types (distinguished by their different colours) are present side by side.

(b) Heterokaryons derived from two strains differing in certain measurable phenotypic characters (e.g. growth-rate, pigmentation, spore density) can be detected by re-extracting both homokaryotic parental strains from single conidiophores.

2. Complementation. (a) In species with multinucleate spores, heterokaryons derived from two parents, each with a different spore colour, can be distinguished if conidia with wild-type spore colouration are produced.

(b) In mixed cultures composed of two homokaryotic strains having (i) different nutritional requirements or (ii) different morphology, heterokaryons arising from them can be detected if they (i) can grow on a minimal medium lacking both of the specific nutrients required by the homokaryotic components, or (ii) arise as single hyphæ or sectors which grow at a different rate to that of either parent. These are usually referred to as "balanced heterokaryons".

Our proof of heterokaryosis (method I $(a)$ ), which involves the segregation of spores with different colours in single conidiophores, is based solely on visual evidence but it is possible that heterokaryons are formed so infrequently by some strains that mixed conidial heads may be overlooked. It is also possible, but we think improbable, that within a heterokaryotic mycelium nuclei of the same genotype might associate and remain together so that the chance of different genomes occurring in the same conidiophore would be remote. Where species with multinucleate spores are involved (method $2(a)$ ), however, the observation of conidial heads with wild-type colouration can be very misleading; conidial heads composed entirely of spores with the wild-type colour which were obtained from mixed cultures of two 

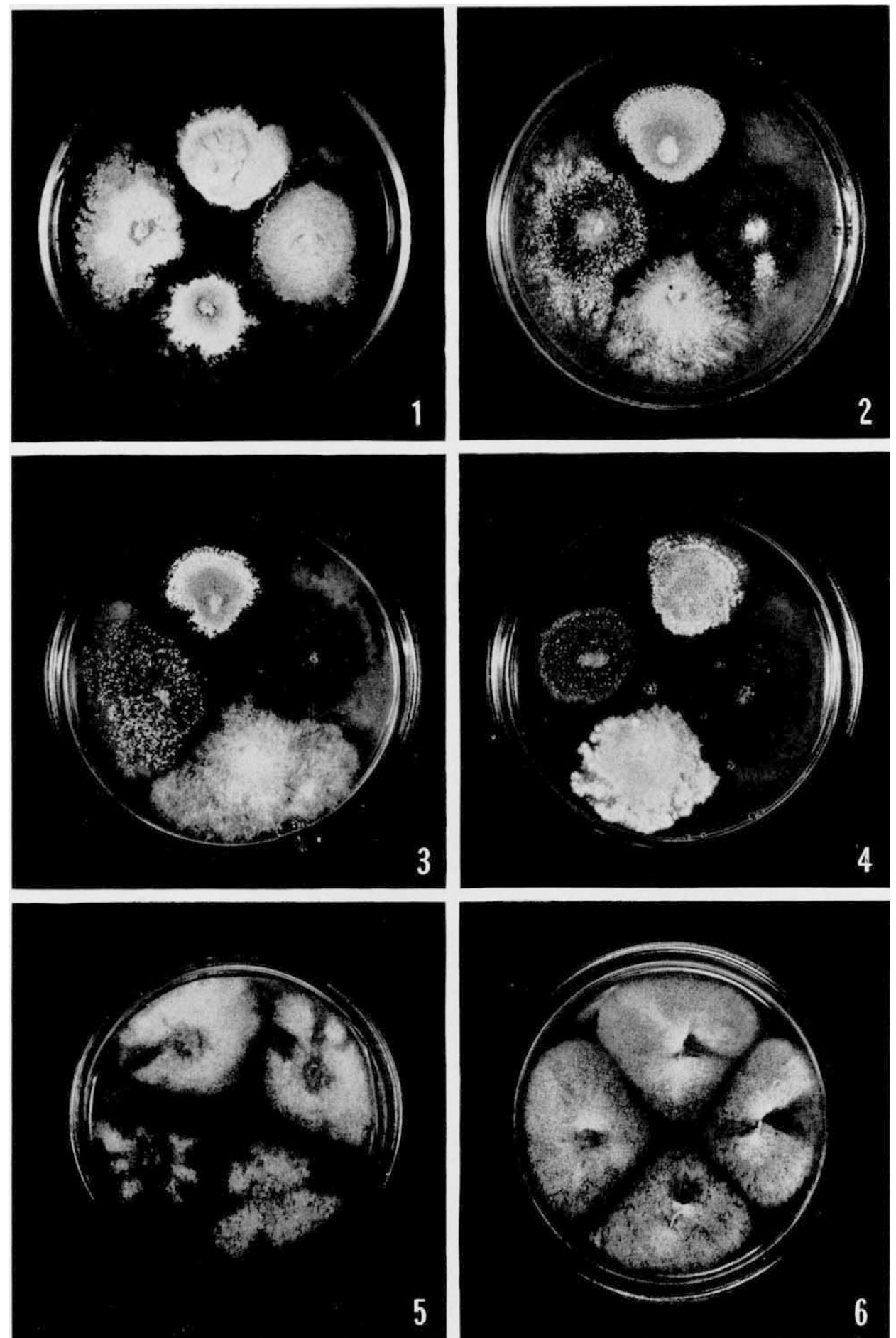

FIGs. 1-4.- - Sixteen-day-old cultures of $A$. nidulans var. echinulatus strain 8 (top), A. quadrilineatus strain 22 (left), A. nidulans strain 1 (right) and $A$. nidulans var. acristatus strain 17 (bottom) growing on (1) MM, $p \mathrm{H} 7 \cdot 0$, (2) $\mathrm{PM}, p \mathrm{H}_{7} \cdot 0$, (3) $\mathrm{PM}, p \mathrm{H}_{5} 6$ and (4) $\mathrm{PM}, p \mathrm{H}_{4} \cdot 4$. Plates show the variation encountered in the nidulans group and the different responses to changes in medium. They also show the usual increase in sporulation resulting from the use of $\mathrm{PM}$ and lowering the $p \mathrm{H}$, strain $\mathrm{I} 7$ being one of the very few exceptions. All heterokaryon tests were carried out on PM with a $p \mathrm{H}$ slightly below that illustrated in fig. 3 .

FIGS. 5 and 6.--Strains 4 and 13 and their spore colour variants growing together in all combinations on (5) PM, $p \mathrm{H}_{5.2}$ and (6) 2 per cent. malt, $p \mathrm{H}_{5}^{\circ} \mathrm{o}$ for determining their heterokaryon compatibility and frequencies of mixed conidial heads. Plate show the superiority of PM over malt agar as a sporulation medium. 

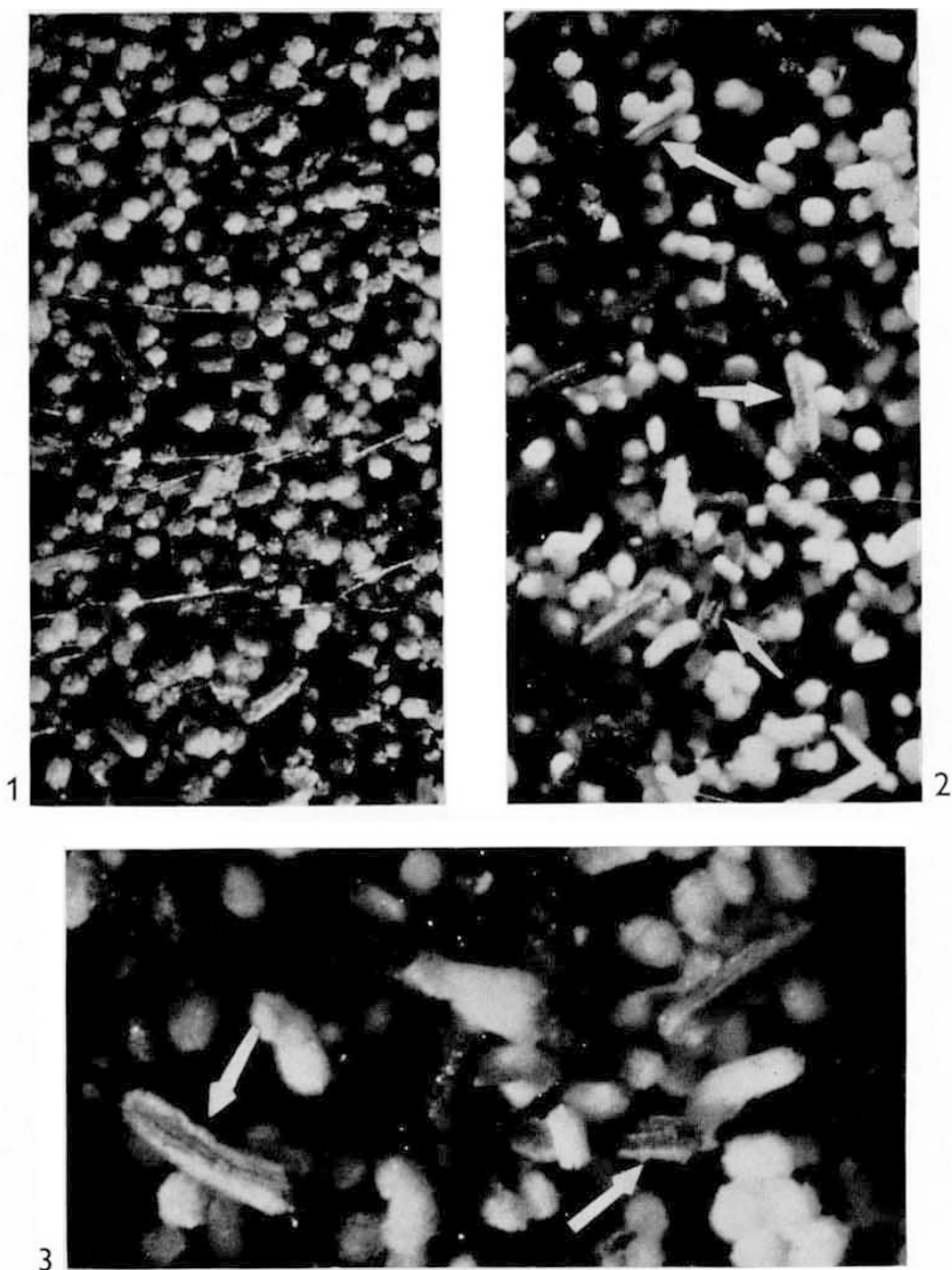

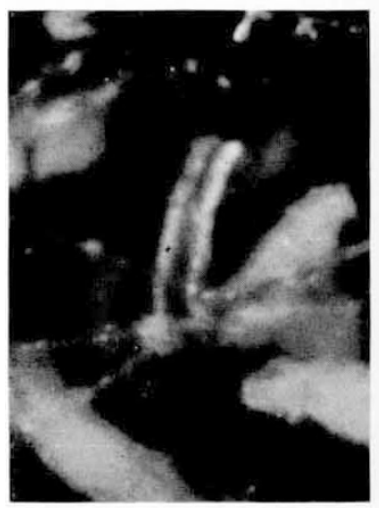

4

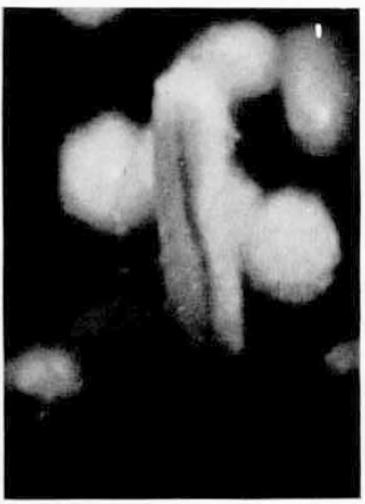

5

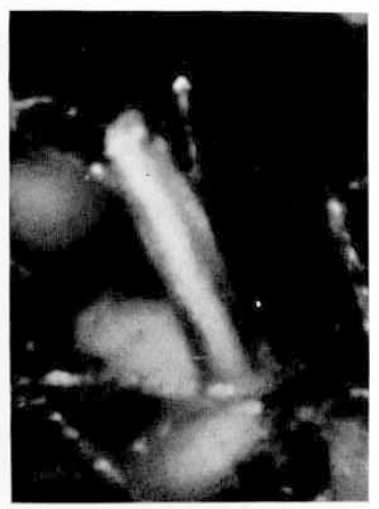

6

FIGs. 1-3.-Conidial heads in a mixed culture of 1 . nidulans strains 2 and $2 \mathrm{w}$ showing (I) density at centre of colony $(\times 40)$, (2) density at edge of colony $(\times 40)$, and $(3)$ density at edge of colony $(\times 100)$. Arrows indicate mixed conidial heads.

Fras. 4, 5 and 6.-Typical mixed conidial heads of $A$. nidulans showing (4) chains of green and yellow sporcs $(5$ and 6$)$ chains of green and white spores. 
different spore-colour mutants of Aspergillus fonseccus have, in some instances, been found to be wild-type in phenotype only since, on progeny testing, only one of the mutant parents was recovered (Raper and Fennell, I953). Ishitani and Sakaguchi (1956) obtained similar results with other Aspergilli and found also that a yellow-spored mutant of $A$. soje developed yellow-green spores if grown on agar containing culture filtrates of other species of Aspergillus as well as of a whitespored strain of the same species. This suggests that some strains produce a substance, or substances, which can be absorbed into the mycelium of another strain and can be transported in the cytoplasm to the site of conidium formation where it affects the pigmentation of the spores. Presumably, whether the wild-type colouration is produced depends on the nature of both the donor and the recipient strains. These same authors have observed green $A$. soje-like conidial heads in mixed cultures of a yellow-spored $A$. soje and a host of other species and genera but in view of their own tests with culture filtrates, any inference that heterokaryons were formed between species and genera is an extremely misleading one.

It is equally misleading to assume that the absence of spores with wild-type colouration proves automatically the absence of heterokaryons since different mutations affecting spore colour need not necessarily complement each other to give phenotypically wild-type conidial heads. Hence, it is possible that Raper and Fennell's (1953) data on $A$. fonsecous which indicate that mutant strains vary greatly in their capacities to form heterokaryons may, in some cases, reflect not the ability of isolates to heterokaryotise but simply their ability to complement each other and produce spores with wild-type colouration. When using species with multinucleate spores as test organisms, therefore, the choice of spore-colour mutants seems to be critical and in any event it would appear advisable to progeny-test (method I (b)) any conidial heads with the wild-type colour from presumptive heterokaryons before the heterokaryotic nature of those spores can be assured. If, however, spores with wild-type colouration occur alongside both mutant spore-types in the same conidial head, evidence for heterokaryosis is based not only on complementation (method $2(a)$ ) but also on segregation (method I $(a)$ ) and such a combination of different criteria must provide undeniable proof that the component strains are heterokaryon compatible.

Two different criteria (methods I $(b)$ and $2(b)$ ) were used in series by Jinks (1952a) to investigate wild heterokaryotic isolates of Penicillium spp. After sampling conidia from some wild strains he obtained two distinct types of asexual progeny which differed from each other and from the parent heterokaryon in their rate of growth. By inoculating the two components together and sampling single hyphal tips from the colony so formed, he then recovered the resynthesised heterokaryon which could be recognised since its rate of growth approximated very closely to that of the original wild 
heterokaryon. This technique provides unambiguous proof of heterokaryosis and should be suitable particularly for detecting wild heterokaryotic isolates or for testing the compatibility of different wild-type strains without first introducing gene mutations which might conceivably effect their ability to heterokaryotise.

Most work done since $195^{2}$ has used the criterion of complementation as described in method $2(b)$, but when using strains with nutritional requirements or testing for "balanced heterokaryons" and meiotic or mitotic recombinants, a heavy selection pressure in favour of the heterokaryon is applied. This technique may be important as a mechanism for producing new strains of imperfect fungi with applications in industrial microbiological work, in which case no comparison or correspondence with events under natural conditions is presumably intended. Such a selection pressure should be relevant and of biological importance under conditions in which heterokaryosis can either confer on otherwise non-infective strains the ability to attack specific host plants or endow the heterokaryon with the ability to grow well on substrates which are less suitable for the homokaryons. However, it is doubtful whether strains differing by many major genes for biochemical requirements would, in fact, be involved to any great extent in nature.

"Balanced heterokaryons" are usually recognised only if they show an improvement in growth over the component homokaryons. Unless other morphological characters are considered, therefore, it must be difficult to distinguish those heterokaryons, should they occur, having a rate of growth which is the same as the parental homokaryotic strains. Likewise, if two mutant strains with different nutritional requirements do not complement each other and are thus unable to grow on MM, they will be overlooked. It seems also that the compatibility as well as the complementarity of different mutants depends on the nature of the nuclear gene mutations. For example, Buxton and Ward's (1962) data on heterokaryosis in Fusaria suggest that the major gene mutations used in an attempt to produce stable heterokaryons had more effect on heterokaryon compatibility than any of the naturally occurring differences which might have been present in the wild strains.

In view of the technical problems inherent in each of the different methods, caution is required when extrapolating from laboratory data to possible events under natural conditions. This applies especially where a single isolate is used as the test strain and all heterokaryons are produced by using mutants derived from that one isolate. It seems, however, that of the various techniques described, the observation of mixed conidial heads by strains with uninucleate spores, although subject to errors, incurs the fewest disadvantages for five reasons. Firstly, mutations affecting spore-colour are easy to induce and only one such mutation in each isolate is required. Secondly, since only one major gene mutation is needed, the possibility 
of introducing incompatibility genes into the strains is minimisedso far, no spore-colour mutant induced in Birmingham has failed to produce mixed conidial heads with its own wild-type strain. Thirdly, many thousands of conidiophores can be scanned rapidly and mixcd heads among them can be distinguished without much difficulty, even when they occur with a very low frequency. Fourthly, the formation of chains of asexual spores with different colours must be unambiguous evidence for the heterokaryotic condition of the mycelium from which such a conidiophore arose; all mixed heads that have been progeny tested in Birmingham have yielded both homokaryotic parental strains. Finally, by using a minimum of induced nuclear gene mutations, results from laboratory tests are more likely to have some bearing on the behaviour of most isolates in the wild.

It seems clear from this investigation that in the sexual species $A$. nidulans, nuclear and cytoplasmic exchange between different isolates is not prevalent and is perhaps confined to strains of closely allied genotype. If similar barriers to heterokaryon formation exist in fungi having no perfect stage, their opportunities under natural conditions for outbreeding by mitotic recombination must be very restricted.

\section{SUMMARY}

Twenty-five different isolates of Aspergillus nidulans and related species were compared on different media and tested for their ability to form heterokaryons.

When grown on Czapek minimal medium (MM) containing New Zealand agar there were large differences between strains in rate of growth, sexuality and sporulation, but when Spanish agar was used in $\mathrm{MM}$, isolates could not be distinguished so easily, and on Plunkett medium or 2 per cent. malt agar many of the differences disappeared. Production of asexual spores by most isolates was increased substantially on any medium by lowering the $p \mathrm{H}$, the best results being obtained on Plunkett medium.

Each wild isolate formed heterokaryons with the spore-colour mutants derived from itself but few examples of inter-strain, and none of inter-species, compatibility were found. Inter-strain compatibility was confined to strains from the same locality but even these formed heterokaryons much more frequently with their own sporecolour mutants than they did with those derived from the other strains.

This heterokaryon test has advantages as a taxonomic tool and as a means of determining strain and species relationships. The significance of our findings for the outbreeding, gene recombination and variation of filamentous fungi is discussed.

Acknowledgments. - I am indebted to Dr J. L. Jinks for his advice and criticism during this investigation and in the preparation of the report, to $\mathrm{Mr} \mathrm{K}$. B. Parry and Miss M. Leighton for assistance with the photography and to Dr Irene Wilson, 
Dr D. Park, Dr R. Cooke and Mr J. Croft who supplied some of the strains. I also wish to thank $\mathrm{Mr}$ J. J. Elphick for identifying some of the Birmingham isolates and for his helpful correspondence.

\section{REFERENCES}

BUXTON, E. W., AND WARD, VALERIE. 1962. Genetic relationships between pathogenic strains of Fusarium oxysporum, Fusarium solani, and an isolate of Nectria haematococca. Trans. Brit. Mycol. Soc., 45, 26I-273.

DHillon, T. S., GARber, E. D., AND WYTTENBach, Ellen G. I961. Genetics of phytopathogenic fungi. VI. Heterokaryosis involving Gibberella fujikuroi and formae of Fusarium oxysporum. Can. F. Bot., 39, 785-792.

GARBER, E. D., WYTTENBACH, ELlen G., AND DHILloN, T. S. I96I. Genetics of phytopathogenic fungi. V. Heterokaryons involving formae of Fusarium oxysporum. Am. F. Bot., 48, 325-329.

ISHITANI, C., AND SAKAGUCHI, K. I956. Hereditary variation and genetic recombination in Koji-molds. V. Heterokaryosis. 7. Gen. Appl. Microbiol., 2, 345-400. JINKs, J. L. I952a. Heterokaryosis in wild Penicillium. Heredity, 6, 77-87.

JINKs, J. L. 1952b. Heterokaryosis : a system of adaptation in wild fungi. Proc. Roy. Soc., B, 140 , 105-145.

KAFER, ETtA. 1958. An 8-chromosome map of Aspergillus nidulans. Adv. Genet., $9,105-145$.

PLUNKETT, B. E. 1953. Nutritional and other aspects of fruit body production in pure cultures of Collybia velutipes (Curt.) Fr. Ann. Bot., N.S. 17 (66), 193-217. pontecorvo, G. 1953. The genetics of Aspergillus nidulans. Adv. Genet., 5, 14 1-238. Pontecorvo, G. 1956. The parasexual cycle in fungi. Ann. Rev. Microbiol., Io, 393-400.

RAPER, K. B., AND fENNELl, DOROThy L. 1953. Heterokaryosis in Aspergillus. $\mathcal{F}$. Elisha Mitchel Sci. Soc., 69, I-29.

ROPER. J. A. 1962. Genetics and microbial Classification. Symp. Soc. Gen. Microbiol., I2, $270-288$.

taschdjian, Claire L., AND MUSKatblitt, E. 1955. Hyphal fusion between Trichophyton tonsurans variants as an indication of species relationship. Mycologia, $47,339-343$.

Wilson, J. F., Garnjobst, LAURA, AND TATUM, E. L. 196r. Heterokaryon incompatibility in Neurospora crassa: micro injection studies. Am. F. Bot., 48, 299-305. 